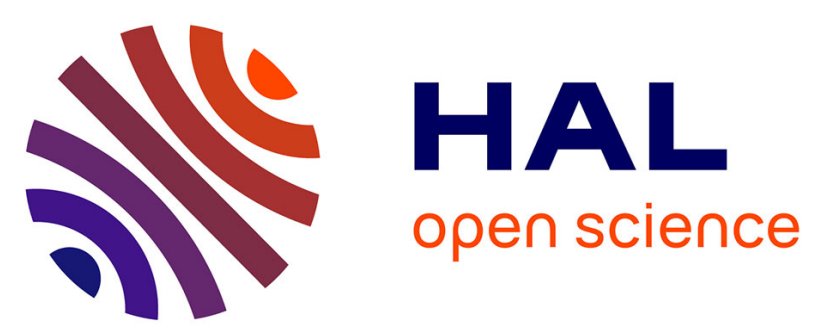

\title{
Long-term in vivo performances of polylactide/iron oxide nanoparticles core--shell fibrous nanocomposites as MRI-visible magneto-scaffolds
}

Hussein Awada, Saad Sene, Danielle Laurencin, Laurent Lemaire, Florence Franconi, Florence Bernex, Audrey Bethry, Xavier Garric, Yannick Guari, Benjamin Nottelet

\section{To cite this version:}

Hussein Awada, Saad Sene, Danielle Laurencin, Laurent Lemaire, Florence Franconi, et al.. Long-term in vivo performances of polylactide/iron oxide nanoparticles core-shell fibrous nanocomposites as MRIvisible magneto-scaffolds. Biomaterials Science, In press, 9, pp.6203-6213. 10.1039/d1bm00186h . hal-03324645

\section{HAL Id: hal-03324645 \\ https://hal.science/hal-03324645}

Submitted on 23 Aug 2021

HAL is a multi-disciplinary open access archive for the deposit and dissemination of scientific research documents, whether they are published or not. The documents may come from teaching and research institutions in France or abroad, or from public or private research centers.
L'archive ouverte pluridisciplinaire HAL, est destinée au dépôt et à la diffusion de documents scientifiques de niveau recherche, publiés ou non, émanant des établissements d'enseignement et de recherche français ou étrangers, des laboratoires publics ou privés. 
Long-term in vivo performances of polylactide / iron oxide nanoparticles core-shell fibrous nanocomposites as MRI-visible magneto-scaffolds

Hussein Awada, ${ }^{1,2}$ Saad Sene, ${ }^{2}$ Danielle Laurencin, ${ }^{2}$ Laurent Lemaire, ${ }^{3}$ Florence Franconi ${ }^{3}$, Florence Bernex,${ }^{4,5}$ Audrey Bethry, ${ }^{1}$ Xavier Garric,${ }^{1}$ Yannick Guari, ${ }^{2}$ and Benjamin Nottelet ${ }^{1 *}$

${ }^{1}$ IBMM, Univ Montpellier, CNRS, ENSCM, Montpellier, France.

${ }^{2}$ ICGM, Univ Montpellier, CNRS, ENSCM, Montpellier, France.

${ }^{3}$ Micro \& Nanomédecines Translationnelles-MINT, UNIV Angers, INSERM U1066, CNRS

UMR 6021, Angers, France ; PRISM Plate-forme de recherche en imagerie et spectroscopie multi-modales, PRISM-Icat Angers, France

${ }^{4}$ [RHEM], BioCampus Montpellier, CNRS, INSERM, Univ Montpellier, Montpellier, France

${ }^{5}$ IRCM, U1194 INSERM, Univ Montpellier, Montpellier, France

*Correspondence: B.N: Benjamin.Nottelet@umontpellier.fr 


\begin{abstract}
There is a growing interest in magnetic nanocomposites in biomaterials science. In particular, nanocomposites that combine poly(lactide) (PLA) nanofibers and super paramagnetic iron oxide nanoparticles (SPIONs), which can be obtained by either electrospinning of a SPIONs suspension in PLA or by precipitating SPIONs at the surface of PLA, are well documented in the literature. However, these two classical processes yield nanocomposites with altered materials properties, and their long-term in vivo fate and performances have in most cases only been evaluated over short periods of time. Recently, we reported a new strategy to prepare welldefined PLA@SPIONs nanofibers with a quasi-monolayer of SPIONs anchored at the surface of PLA electrospun fibers. Herein, we report on a 6-month in vivo rat implantation study with the aim of evaluating the long-term magnetic resonance imaging (MRI) properties of this new class of magnetic nanocomposites, as well as their tissue integration and degradation. Using clinically relevant T2-weighted MRI conditions, we show that the PLA@SPIONs nanocomposites are clearly visible up to 6 months. We also evaluate here by histological analyses the slow degradation of the PLA@SPIONs, as well as their biocompatibility. Overall, these results make these nanocomposites attractive for the development of magnetic biomaterials for biomedical applications.
\end{abstract}

Keywords: hybrid biomaterial, iron oxide nanoparticles, poly(lactide) nanofibers, medical resonance imaging (MRI), histology, implantation, in vivo 


\section{1- Introduction}

Nanocomposites formed by the association of a polymeric nanostructured phase (e.g. nanoparticles, nanofibers) and inorganic nano-objects (e.g. nanospheres, nanorods) are increasingly being developed to fulfil various needs of modern biomedical applications. This is the case in sensors, actuators, and scaffolds, as well as for theranostic applications. ${ }^{1,2}$ In particular, nanocomposites composed of degradable polymers like the well-known poly(lactide) biopolymer (PLA), and of magnetic nanoparticles, like the widely used super paramagnetic iron oxide nanoparticles (SPIONs), are of particular interest as they gather degradability, biocompatibility, magnetic and photo-thermal properties, and magnetic resonance imaging (MRI) abilities, which makes them biomaterials of choice for theranostic applications. ${ }^{3,4}$ Until recently, such nanocomposites were either prepared as SPION-loaded PLA-nanoparticles, or as SPION-loaded composites including electrospun fibers for tissue engineering applications. These nanocomposites have generally been obtained by processing a mix of polymer/SPION or polymer/SPION precursors, or by growing a layer of SPIONs on preformed polymeric surfaces through precipitation. ${ }^{5}$ The first strategy has been exemplified recently in works involving PLA or polycaprolactone (PCL) scaffolds for faster bone or tissue repair under external magnetic fields, which stimulate cell proliferation and secretion of new extra-cellular matrix. ${ }^{6-9}$ Other authors reported on the use of magnetic polymeric nanofibers obtained by electrospinning for triggered drug delivery assisted by magneto thermal effect, or to monitor the drug release. ${ }^{10-12}$ However, all these synthetic strategies are associated with various drawbacks, including an absence of control over the morphology/repartition of the precipitated/dispersed SPIONs, a decrease of the magnetization at the saturation of SPIONs, ${ }^{7}$ a modification the mechanical properties of the polymer, ${ }^{8,13,14}$ and in the case of degradable polymers, uncontrolled and faster degradation kinetics (eg. 3.5 fold faster in vitro degradation for PCL fibers $v s$. PCL embedding SPIONs). ${ }^{8,15}$ 
To overcome these drawbacks, we have recently reported on a new strategy to prepare PLA@SPIONs nanofibers with a controlled interface: a quasi-monolayer of SPIONs anchored at the surface of PLA electrospun fibers. ${ }^{16}$ Such well-defined magnetic nanocomposites are expected to present major benefits over the existing magnetic polymeric composites reported in the literature: (i) no change in the mechanical and degradation properties of the polymeric phase due to surface immobilization of the inorganic particles (rather than their mixing in the polymer matrix), (ii) enhanced magnetic properties through improved dipolar interactions of the surface anchored superparamagnetic nanoparticles, (iii) enhanced MRI sensitivity, (iv) lower quantities of magnetic nanoparticles for high magnetic properties and therefore lower biocompatibility concerns. Some of these points were confirmed in our proof of concept study. We had demonstrated a preservation of the magnetic properties of the anchored SPIONs that exhibited similar values of the saturation magnetization compared to free SPIONs. We had also proved the in vitro cytocompatibility of the PLA@SPIONs as well as their ability to be visualized in vitro using T2-weighted MRI. Finally, a preliminary in vivo MRI experience had confirmed MRI visualization after 1 week.

This set of data was promising. However, to ensure further development and use of this new class of magnetic nanocomposites, the question of their fate, including their long-term integration and biocompatibility in tissues, and their long-term MRI visibility, is crucial. It is to note that although the question of the so called "acute", short term, biocompatibility of such nanocomposites is generally addressed in the literature, the question of their visibility and biocompatibility over extended periods of time is not. In fact, most studies related to implantable MRI-visible biomaterials embedding SPIONs assess this imaging ability over a limited period ranging from few hours to few days. ${ }^{9,11,17,18}$ 
The aim of this study is therefore to assess these criteria for our newly developed PLA@SPIONs nanofibers thanks to a 6 months in vivo study (Scheme 1). First, results of an in vitro study aiming at evaluating the changes encountered by the nanocomposites during degradation are presented, after which the histological and MRI outcomes of a 6 monthimplantation in rat are discussed. Overall, the results demonstrate the applicability of the PLA@SPIONs nanocomposites as biomaterials for in vivo applications.

\section{2- Experimental}

\section{1- Materials}

Propargyl bromide (80 wt\% in toluene), lithium diisopropylamide (LDA, 2M in THF/heptane/ethylbenzene), 2,2-dimethoxy-2-phenylacetophenone (DMPA, >99\%), tris(2carboxyethyl)phosphine hydrochloride solution (TCEP, 0.5M, pH7), FeOOH (catalyst grade, 30-50 mesh), oleic acid (90\% purity) and $n$-docosane (99\% purity) were purchased from SigmaAldrich. Oleylamine, with an approximate C18 content of 80-90\% (97\%), was purchased from Acros Organics. 12-mercaptododecylphosphonic acid was purchased from Sikemia. Poly(Llactide) $\left(\right.$ PLA $_{100}$ noted PLA in the manuscript, NatureWorks, grade 6201D) was purchased from Cargill Dow LLC (Minnetonka, MN). Absolute ethanol, cyclohexane, methanol, chloroform and acetone (99\% purity) were used as received. Dry THF was obtained by the solvent purification system PureSolve MD5 from Innovative Technology. Fresh diethyl ether $\left(\mathrm{Et}_{2} \mathrm{O}\right)$ was dried over molecular sieves prior to use. 


\section{2- Methods}

Scanning Electronic Microscopy (SEM) and Energy Dispersive X-ray Spectroscopy (EDXS) analyses were performed on a FEI Quanta FEG 200 instrument equipped with an Oxford Instruments Ultim Max SDD $100 \mathrm{~mm}^{2}$ EDX detector. The fibers were deposited on an adhesive carbon film and analyzed under vacuum. The quantification of the heavy elements was carried out with the INCAC software, with a dwell time of $3 \mu \mathrm{s}$.

Some of the samples were also analyzed by SEM on a Zeiss Evo HD15 scanning electron microscope equipped with an Oxford Instruments X-MaxN SDD $50 \mathrm{~mm}^{2}$ EDX detector.

Transmission electron microscopy (TEM) was performed on a JEOL 1200EX transmission electron microscope, using an accelerating voltage of $100 \mathrm{kV}$. Ultrathin sections of the SPIONfunctionalized PLA fibers (30-100 nm in thickness) were obtained using a Leica ultracut ultramicrotome, after setting the sample in an LR-White resin. The thin sections were then placed on carbon-formvar coated copper grids. For each sample, several TEM images of the cross sections were recorded, and only a selection of these is shown here.

Thermal gravimetric analyses (TGA) were performed on a TGA 2 Star $^{\mathrm{e}}$ System, Metler Toledo at a heating rate of $10^{\circ} \mathrm{C} \cdot \mathrm{min}^{-1}$ under air.

Size exclusion chromatography (SEC) analyses were performed using a Shimadzu Prominence system equipped with a RID-20A refractive index signal detector coupled to a SPD-20A UV/VIS detector and to a PLgel MIXED-C guard column (Agilent, $5 \mu \mathrm{m}, 50 \times 7.5 \mathrm{~mm}$ ) and two PLgel MIXED-C columns (Agilent, $5 \mu \mathrm{m}, 300 \times 7.5 \mathrm{~mm}$ ). Samples (5 mg) were dissolved in THF ( $1 \mathrm{~mL})$ and filtrated through a $0.45 \mu \mathrm{m}$ PTFE Millipore filter. The mobile phase was THF, 
and the flow rate $1.0 \mathrm{~mL} \cdot \mathrm{min}^{-1}$. The injection volume was $100 \mu \mathrm{L}$. The average molecular weights and dispersities (Đ) were calculated using polystyrene (PS) standards.

Inductively coupled plasma-optical emission spectrometry (ICP-OES) analyses were performed by L. Koeller at the AETE-ISO platform, OSU-OREME/Université de Montpellier, using a iCap 7400 Duo Thermofisher equipment. PLA@SPIONs samples were digested in hot nitric acid prior to dilution for analysis.

\section{Electrospun PLA fibers}

Random electrospun fibers were prepared from a solution of PLA dissolved in chloroform (8 $\mathrm{wt} \%$ ) and collected on a flat collector. The electrospinning parameters were the following: pump rate $2.0 \mathrm{~mL} / \mathrm{h}$, collection time $60 \mathrm{~min}$, voltage $12 \mathrm{kV}$, room temperature, and a $15 \mathrm{~mm}$ gap distance between the needle and the grounded collector.

\section{Functionalization of PLA fibers with phosphonic acid groups}

The functionalization of PLA fibers with phosphonic acid groups was carried out in two steps as previously described by our group. ${ }^{16}$ In brief, PLA fibers were first propargylated in diethyl ether at $-50^{\circ} \mathrm{C}$ by an anionic activation using LDA, followed by the addition of propargyl bromide. In a second step, 12-mercaptododecylphosphonic acid ( $\left.\mathrm{HS}-\left(\mathrm{CH}_{2}\right)_{12}-\mathrm{P}(\mathrm{O})(\mathrm{OH})_{2}\right)$ was grafted on the PLA fibers thanks to a thiol-yne photoaddition reaction under UV between the free alkyne groups present at the surface of the PLA fibers and the thiol functionality of the phosphonic ligand. Detailed synthetic procedures are available in the supporting information. 
Preparation of super magnetic iron oxide nanoparticles (SPIONs)

Oleic acid-functionalized SPIONs were synthesized according to a procedure of the literature. ${ }^{19}$ The detailed synthetic procedure is available in the supporting information.

\section{Preparation of PLA@SPIONs nanocomposites}

The anchoring of the oleic acid-functionalized SPIONs to the surface of the phosphonic acidfunctionalized PLA fibers was carried out according to a ligand exchange procedure recently described by our group. ${ }^{16}$ In short, freshly prepared oleic acid-functionalized SPIONs were suspended in cyclohexane/ acetone $(3: 1 \mathrm{v}: \mathrm{v})$ and were sonicated for $2 \mathrm{~h}$ under argon. PLA meshes were then immersed in the suspension of SPIONs and the ligand exchange was let to proceed for $18 \mathrm{~h}$ at room temperature under argon in the dark. SPION-functionalized meshes were then cleaned by successive washing and sonication steps in a mixture of cyclohexane and acetone, before drying under argon atmosphere. The SPION-functionalized meshes were stored in the fridge. More detailed synthetic procedures are available in the supporting information.

\section{In vitro degradation}

Samples were immersed in $20 \mathrm{~mL}$ phosphate buffer saline (PBS 1X) in test tubes, and maintained at $37^{\circ} \mathrm{C}$ under orbital shaking in a thermostatic incubator. The PBS was replaced weekly in order to keep the $\mathrm{pH}$ of the solutions stable. At defined time points, samples were collected for analyses (TEM, SEM/EDX, ICP-OES). In parallel, the evolution of molecular weight was measured by SEC and the mass loss of the samples was calculated according to the following equation:

$$
\text { Mass loss }(\%)=\frac{m_{0}-m_{d}}{m_{0}} \times 100
$$

with $\mathrm{m}_{0}$ the initial mass and $\mathrm{m}_{\mathrm{d}}$ the dry mass at the defined time point.

For degradation in water, the same protocol was followed by replacing PBS by ultrapure water. 


\section{3- Implantation of PLA@SPIONs nanocomposites}

Animal care and use were in accordance with the regulations of the French Ministry of Agriculture and approved by the Pays de la Loire Ethics in Animal Experimentation Committee under project number 01858.03. Animals were housed in a controlled and pathogen-free environment, with free access to food and water, at the University animal facility (SCAHUAngers, France). The implantation of the PLA fibers and of PLA@SPIONs fibers within the peritoneal cavity was performed on isoflurane anesthetized female Sprague Dawley rats (220$250 \mathrm{~g}, \mathrm{n}=4$ ) (continuous flow of air $-0.8 \mathrm{~L} / \mathrm{min}-2 \%$ isoflurane (Piramal Healthcare, UK Limited, Northumberland, UK), pre-treated with a $30 \mu \mathrm{g} / \mathrm{kg}$ subcutaneous injection of Vetergesic ${ }^{\circledR}$ (buprenorphin, Sogeval, France). Briefly, after a median incision of the abdominal wall, a $2 \mathrm{~cm}^{2}$ piece of PLA fibers or of PLA@SPIONs fibers scaffold was placed on each side of the incision and fixed to the wall with 4 nylon $7 / 0$ stitches.

\section{4- Magnetic Resonance Imaging}

Following implantation, in vivo MR imaging on anaesthetized animals (isoflurane) was performed at defined times points ( 1 week, 2 weeks, 1 month, 3 months and 6 months). MR imaging was performed on a Bruker Biospec 70/20 system operating at a magnetic field of 7T (Bruker, Wissembourg, France) using a 72-mm diameter birdcage resonator and a respiratory triggered RARE sequence (effective repetition time TR $2000 \mathrm{~ms}$; rat breathing rate 4050/min; effective echo time TEeff $=23 \mathrm{~ms}$; RARE factor $=8$; FOV $=55 \mathrm{~mm} \times 55 \mathrm{~mm}$; matrix $256 \times 256$, slice thickness $=1 \mathrm{~mm}$ and 8 accumulations).

A movie (supporting information Movie S1) of the scaffolds was obtained from the dataset acquired 2 weeks after implantation by superimposition of two maximum intensity projections, one corresponding to the anatomical data (gray), and one obtained after contour delineation of the scaffolds and background suppression (orange). 
2.5- Explantation, histopathology preparation and tissue evaluation

One rat was euthanized 3 months after implantation and the 3 others were euthanized at 6 months post-implantation. Full-thickness sections of the abdominal wall, including all scaffold sites with ca. $1 \mathrm{~cm}$ of surrounding tissue were recovered, fixed in $10 \%$ formalin before paraffin embedding, slicing and staining with hematoxylin-eosin-suffran (HES) to assess cellular reactions of the tissue. Perls staining was performed with Neutral Red as a counter stain (RHEM, Montpellier). This staining allows to highlight the possible cellular uptake of iron $\mathrm{Fe}^{3+}$ from the scaffold in the surrounding tissues. Analysis was done using a Zeiss Axioplan microscope and images were acquired using an Axiocam digital camera and used with AxiovisonLE software.

The host-tissue morphologic response to the presence of the scaffolds was assessed. Intensity and distribution of cellular infiltration, multinucleated giant cell presence, vascularization, and reorganization of the collagen fibers were evaluated with semiquantitative methods. The presence of multinucleated giant cells, connective tissue and neovascularization were scored according to a semi-quantitative histopathologic analysis scale (Table S1 and Table S2), adapted from methodologies reported in the litterature..$^{20,21}$ The scoring was graded by one senior pathologist, blinded to the origin of the samples.

\section{3- Results and discussion}

\subsection{Preparation and degradation of PLA@SPIONs nanofibers}

PLA@SPIONs nanofibers were prepared according to our recently-reported procedure (Scheme 1). ${ }^{16}$ Random PLA mesh nanofibers were first functionalized with phosphonic acid groups following a two step-procedure involving the propargylation of PLA nanofibers followed by thiol-yne photoaddition of 12-mercaptododecylphosphonic acid. In a next step, oleic acid-functionalized SPIONs with a diameter around $18 \mathrm{~nm}$ (Figure S1), were anchored 
onto the PLA nanofibers, thanks to the ligand exchange taking place between the carboxylic groups present at the surface of the SPIONs and the phosphonic acid groups present at the surface of the PLA nanofibers. This exchange was driven by the stronger affinity of the phosphonic group for metal oxides compared to carboxylic groups, that allows the oleic acid shell to be removed from the surface of the SPIONs. ${ }^{22}$

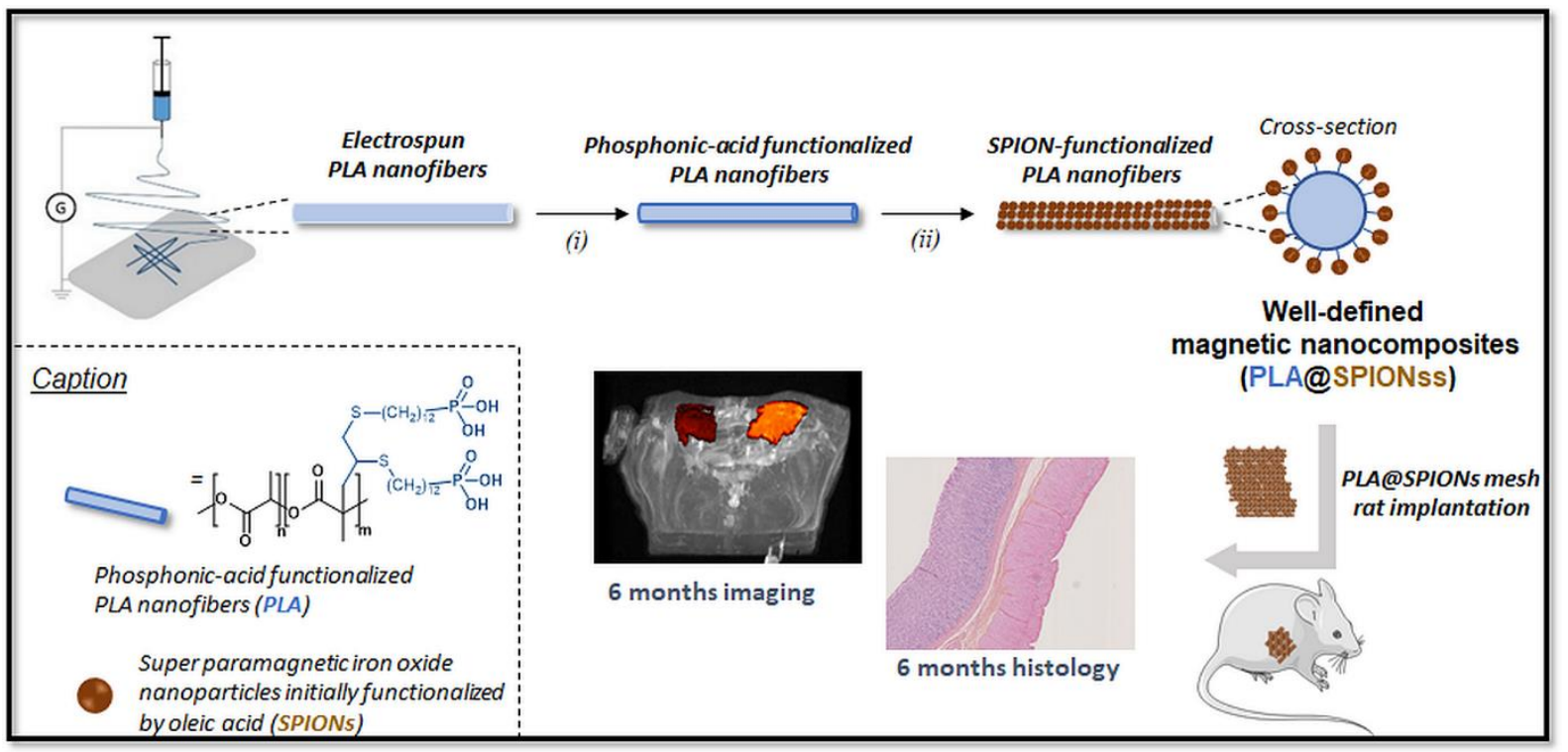

Scheme 1. Schematic representation of the synthetic strategy followed for the preparation of well-defined magnetic nanocomposites PLA@SPIONs and overview of the in vivo studies performed. i) functionalization of the PLA nanofibers surface with phosphonic acid groups and ii) ligand exchange procedure to anchor SPIONs at the surface of the phosphonic-acid functionalized PLA fibers.

PLA@SPIONs meshes were characterized by SEM and TEM (Figure 1). No degradation of the PLA fibers was observed during the grafting process (Figure 1a). SEM images also confirmed the absence of SPION aggregates, which confirmed the efficiency of the cleaning procedure (Figure 1a inset). As expected based on our previous study, TEM images confirmed the presence of SPIONs anchored at the surface of the PLA fibers forming a homogeneous and 
quasi-complete coverage (Figure 1b). This layer of SPIONs corresponds to ca. $8 \mathrm{wt} \%$ of iron oxide in the nanofibrous composites, as determined by TGA analysis. ${ }^{16}$
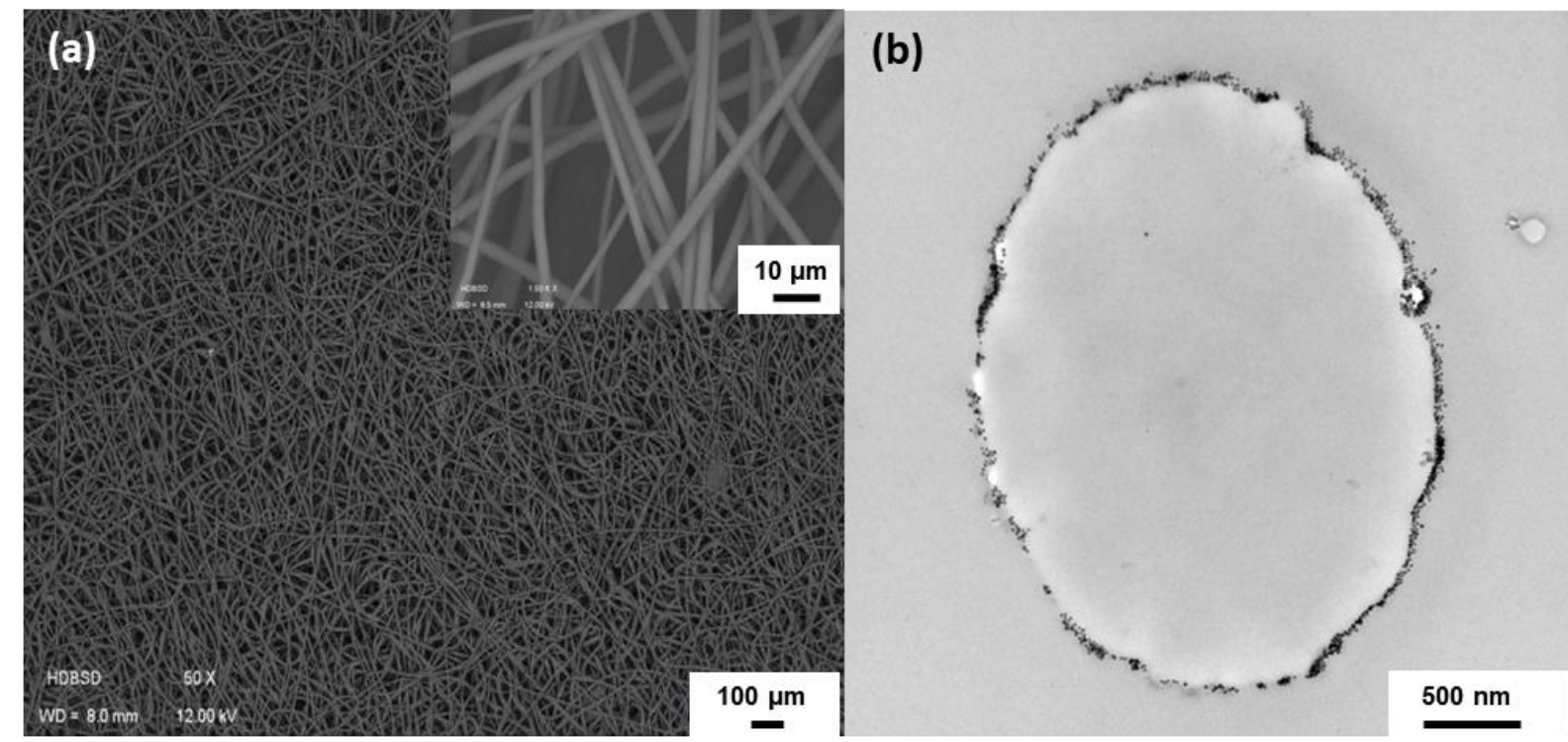

Figure 1. Morphological analysis of PLA@SPIONs. (a) SEM image of a mesh of fibers, (b) cross sectional TEM image of a single fiber

The degradation of PLA@SPIONs scaffolds was then assessed under physiologically relevant conditions using phosphate buffer saline (PBS, $\mathrm{pH} 7.4,37^{\circ} \mathrm{C}$ ). The aim of this in vitro evaluation was to determine the durability of the SPIONs' anchoring (Figure 2). First, and as seen on the pictures, PLA fibers did not show morphological alterations during this one-month follow-up. This is not surprising considering the nature of the PLA 100 phase, also referred as PLLA, that was used here, which is known to degrade very slowly under these conditions. ${ }^{23}$ This was further confirmed by following the samples' mass over time (Figure S2): no significant mass loss was observed for PLA@SPIONs fibers, suggesting the absence of macroscopic degradation. Second, a clear redistribution of the SPIONs was observed during the PBS immersion process, with areas of higher concentration and areas of lower concentration of SPIONs appearing at the surface already after 1 week (Figure 2b). After 1 month, only a few 
areas of the PLA surface were still covered with SPIONs, most of them being aggregated and forming very large clumps (Figure 2c).

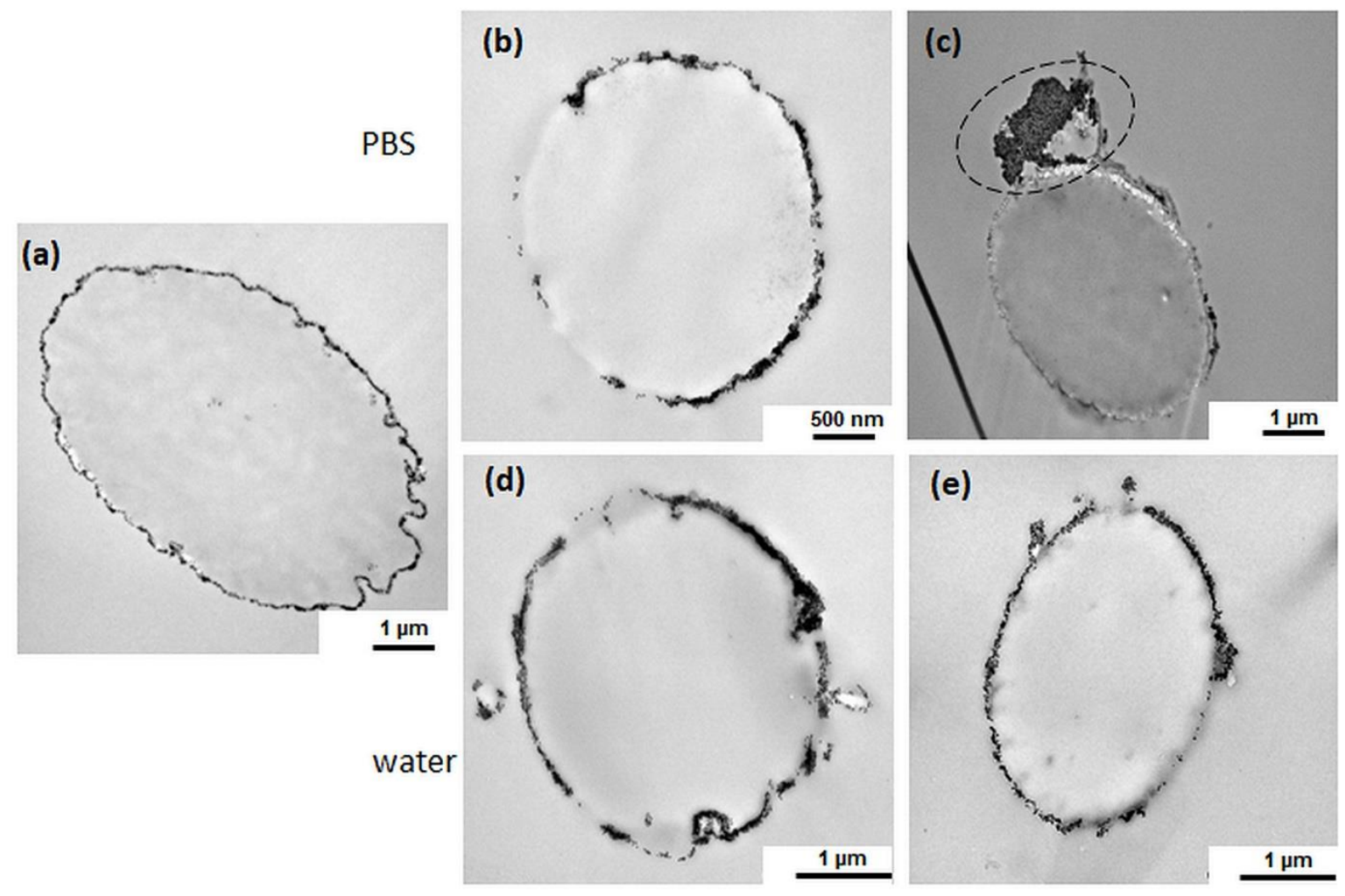

Figure 2. In vitro degradation of PLA@SPIONs. TEM images of PLA@ SPIONs nanofibers before (a) and after degradation in PBS solution $\left(37^{\circ} \mathrm{C}, \mathrm{pH} 7.4\right) 1$ week (b) and 1 month (c) or in water $\left(37^{\circ} \mathrm{C}\right)(\mathrm{d}) 1$ week and (e) 1 month.

This evolution had not been studied in our previous work, in which only samples after 3 days of immersion in PBS had been used to perform an EDXS analysis, which had suggested that the atomic percentage of Fe element was constant for this duration. The current study actually demonstrates a rearrangement and a redistribution of the SPIONs around the fibers over time, with a progressive loss of coverage of the fibers. Two factors can explain this phenomenon. First, the degradation of the PLA chains at the surface of the fibers, that could lead to a loss of anchoring for the SPIONs. However, SEC analyses showed only limited decrease of the number 
average molecular weight over 1 month (15\% Mn decrease) and, as previously mentioned, no macroscopic mass loss was detected. These two results taken together do not confirm this first hypothesis, that would require the release of leachable oligomers (Figure S2). Second and more likely, due to the large excess of phosphate ions in the buffer, the competition between phosphate/phosphonate groups at the surface of the SPIONs can lead to the reversible loss of the coordination bond, and further to the clustering of the SPION particles, which become "connected" to each other through phosphate bridges. This second hypothesis would be in line with other studies of phosphonate-grafted biomaterials, which report a partial surface substitution of phosphonate anchors by phosphate ions of simulated body fluid. ${ }^{24}$

To further investigate this point, we performed additional studies on PLA@SPIONs fibers immersed either in deionized water or in PBS, and compared the evolution of the samples after 1 week and 1 month immersion by TEM imaging (Figure 2), EDXS and ICP-OES analyses. In TEM, the redistribution of the SPIONs previously noticed in PBS was not observed in deionized water. Moreover, although the surface of the samples overall became more heterogeneous in nature after 1 month immersion in deionized water, no formation of aggregates was observed (Figure $2 \mathrm{~d}$ and $2 \mathrm{e}$ ). This would be in line with the previous hypothesis, stating that the aggregation of the SPION nanoparticles relies on the presence of phosphate ions in the release medium.

To go one step further, the evolution in the content of Fe and $\mathrm{S}$ elements in the samples after 1 week and 1 month degradation in both media was analyzed, using SEM-EDX analyses. The Fe element is representative of the SPIONs, whereas the S element is characteristic of the 12mercaptododecylphosphonic acid covalently attached at the surface of the PLA fibers. Hence, comparing evolutions in Fe/S ratios by EDXS can indirectly inform on the amount of SPIONs still attached at the surface of the PLA fibers. In our case, EDXS analyses of portions of the fiber surfaces where both elements could be properly detected showed that in both deionized 
water and PBS, the Fe/S ratio decreases over time. Moreover, it was found that the decrease in this ratio between the one week and one month time-points is only slightly more pronounced in PBS compared to deionized water (decrease by a factor of $~ 1.7$ in PBS $v s 1.5$ in pure water, according to EDXS). While the more significant decrease in PBS can be explained by a more efficient release of some of the SPIONs from the fiber surface due to the phosphate/phosphonate competition, the fact that both Fe/S ratios globally evolve similarly in both media could be in line with TEM observations, which suggest that some SPIONs remain close to the fiber surface in an aggregated form (Figure 2c). Complementary ICP-OES analyses were also carried out, and confirmed the EDXS analyses by showing that the amount of Fe present at the surface of the fibers decreased in the same range upon exposure to PBS for 1 week and 1 month (Figure S3). Therefore, overall, from TEM, EDXS and ICP-OES analyses, it can be concluded that in the case of PBS, some particles remain aggregated at the viscinity of the fibers' surface, despite having been removed from the initial surface coating.

This set of data illustrates the "dynamic" nature of the SPIONs' ligation with phosphonic groups, which, despite their strong affinity for the iron oxide phase, can be partially replaced by phosphates. It is however important to note that these results obtained under in vitro conditions (including mild orbital shaking) might be far from the in vivo scenario where surrounding tissues may impede SPIONs redistribution. To evaluate this point, a long-term rat implantation was therefore conducted.

\subsection{In vivo MRI visualization of PLA@SPIONs nanofibers}

The ability to visualize the PLA and PLA@SPIONs nanofibers under MRI over extended periods of time was evaluated. Samples of nanofibrous materials (Figure S4A) were cut and implanted in four Sprague Dawley rats. The two types of scaffolds were implanted in each rat 
with one scaffold being placed on each side of the abdominal wall of the animals. At defined times points, rats were anaesthetized and imaged using a standard T2-weighted spin echo sequence and a pre-clinical MR scanner (Figure S4B). At 1-week post-implantation, a clear hyposignal was observed for the PLA@SPIONs scaffolds due to the SPIONs anchored at the surface of the PLA fibers and acting as superparamagnetic contrast agents (Figures 3A\&A', beige arrows). In contrast, the bare PLA scaffolds could hardly be detected (Figures 3A\&A', white arrows). The same observations were made 2 weeks (Figure 3B) and 1 month (Figure 3C) after implantation, with no evidence of signal loss. This is further illustrated by the MRI 3D-reconstruction of the abdominal cavity of a rat at 2 weeks, showing after image treatment the PLA scaffold in orange, whereas the PLA@SPIONs scaffold appears in dark red in accordance with the hyposignal obtained with T2 agents (Figure S4C \& Movie S1).

Only after 3 months did the hyposignal associated with the PLA@SPIONs nanofibers start to decrease in intensity (Figure 3D). The signal intensity decrease is due to the degradation of the PLA@SPIONs. Indeed, the subsequent internalization of SPIONs in macrophages for their elimination could be confirmed by histological slide sections at 3 and 6 months (Figures 4F \& $5 \mathrm{~F})$.

However, even at 6 months post-implantation, the PLA@SPIONs were easily detected and were still clearly distinct from the surrounding tissues (Figure 3E). This result confirms the ability to visualize these magnetic nanocomposites over extended periods of time in vivo. Interestingly, it also shows that the fast rearrangement and redistribution of the SPIONs that had been witnessed in vitro does not seem to affect the in vivo imaging of these PLA@SPIONs nanofibers. Two hypotheses can be drawn from this observation: either the rearrangement and redistribution of the SPIONs are impeded by the integration of the scaffolds in the tissues, as confirmed by the histological analyses (see next paragraph), or despite this process, the clumps 
that are formed still allow the imaging properties of the PLA@SPIONs nanofibers to be maintained.
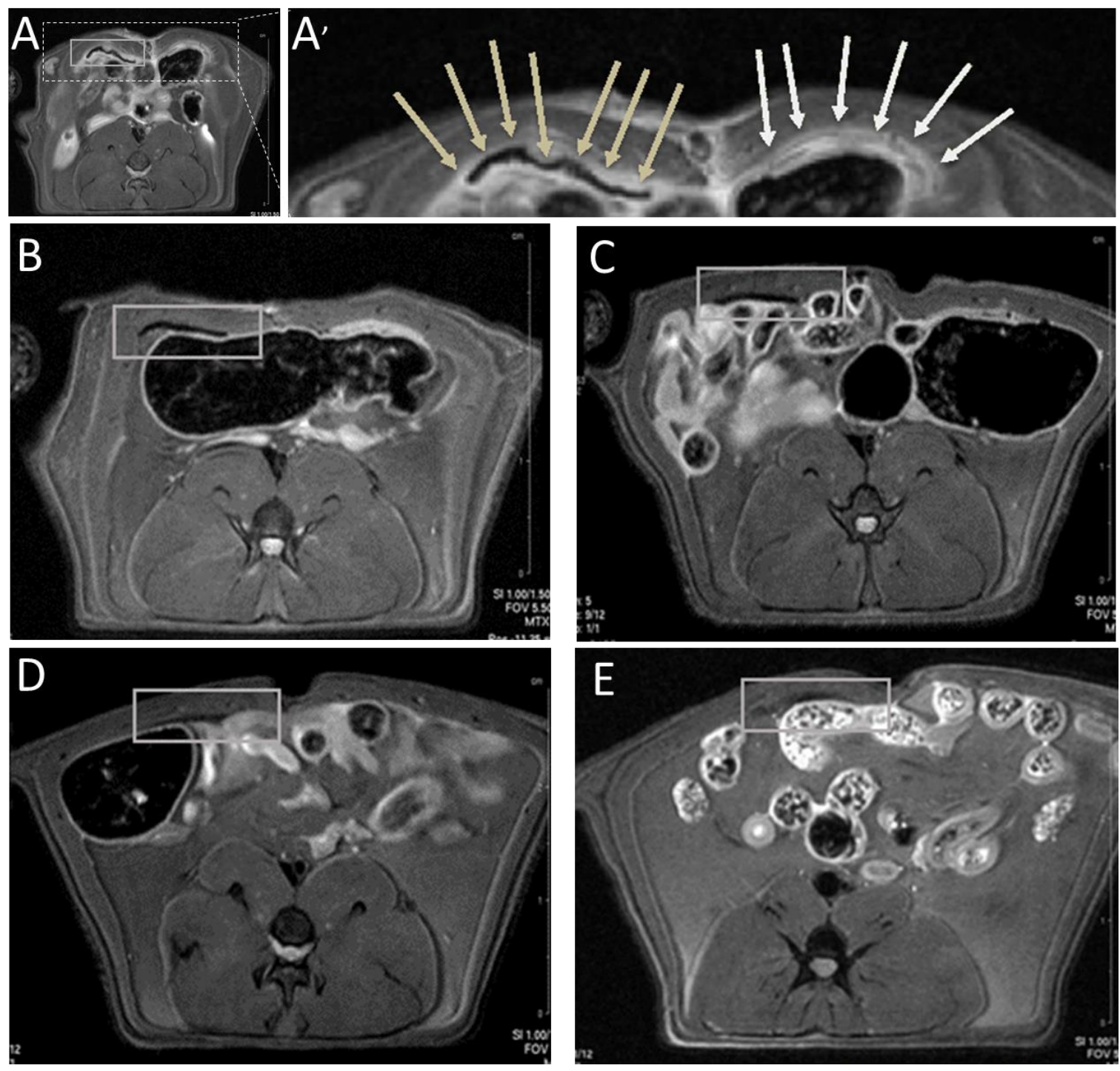

Figure 3. In vivo 6-month follow-up of PLA@SPIONs nanocomposites using magnetic resonance imaging. T2-weighted MR images of PLA@SPIONs nanocomposites implanted in the abdominal cavity of rats for (A) 1 week, (B) 2 weeks, (C) 1 month, (D) 3 months and (E) 6 months. (Inset ( $\left.\mathrm{A}^{\prime}\right)$ corresponds to a magnification of the area of interest at 1 week postimplantation, with beige arrows highlighting the PLA@SPIONs scaffolds, and white ones the bare PLA scaffolds). 


\subsection{Histological evaluation of PLA and PLA@SPIONs scaffolds}

The PLA and PLA@SPIONs fibers were studied at 3 months and 6 months post implantation. Typically, a foreign body introduced in a normal environment induces a chronic inflammatory reaction, with a marked increase of various immune cells. Here this reaction, despite moderate, was observed, with immune mononuclear cells such as lymphocytes and macrophages associated with pathognomonic multinucleated giant foreign-body type cells. Both PLA and PLA@SPIONs scaffolds induced this chronic foreign-body inflammatory response, with a typical host versus graft foreign body granulomatous reaction. It was seen at the HES staining in the peritoneal serosa on the muscle, and allowed the scaffolds to be demarcated. Besides HES staining, Perls staining was also done as this coloration is often used to highlight iron positive macrophages, and allows the fate of SPIONs in various implantation sites to be assessed..$^{25,26}$

When looking in more detail, for the PLA scaffolds at 3 months post-implantation, HES stained slide sections revealed a scaffold well demarcated from the muscle wall, with a moderate chronic inflammatory reaction, but no necrosis (Figure 4A). Various immune cells were observed, mostly macrophages associated to lymphocytes and giant cells. Giant plurinucleated cells showed nuclei concentrated at the cell periphery, and a cytoplasm often filled with 1 to 3 optically white ovoid structures, corresponding to the fibrous materials (Figure 4B). ${ }^{27}$ When present, a few eosinophils were associated with some mast cells. Fibrosis was mild with presence of collagen fibers. For PLA@SPIONs nanocomposites, HES stained sections showed the same moderate chronic inflammatory reaction after 3 months of implantation (Figure 4D \& 4E). The analysis of the quantification and distribution of the cells between PLA and PLA@ SPIONs scaffolds showed no difference between the two materials (Table S1, supplementary data). With the Perls staining no blue coloration was observed with the PLA nanofibers (Figure 4C), whereas for PLA@SPIONs a light blue film around the fibers was revealed in the giant plurinuclear cells, and a blue granular content in the cytoplasm of the macrophages (Figure 4F). 

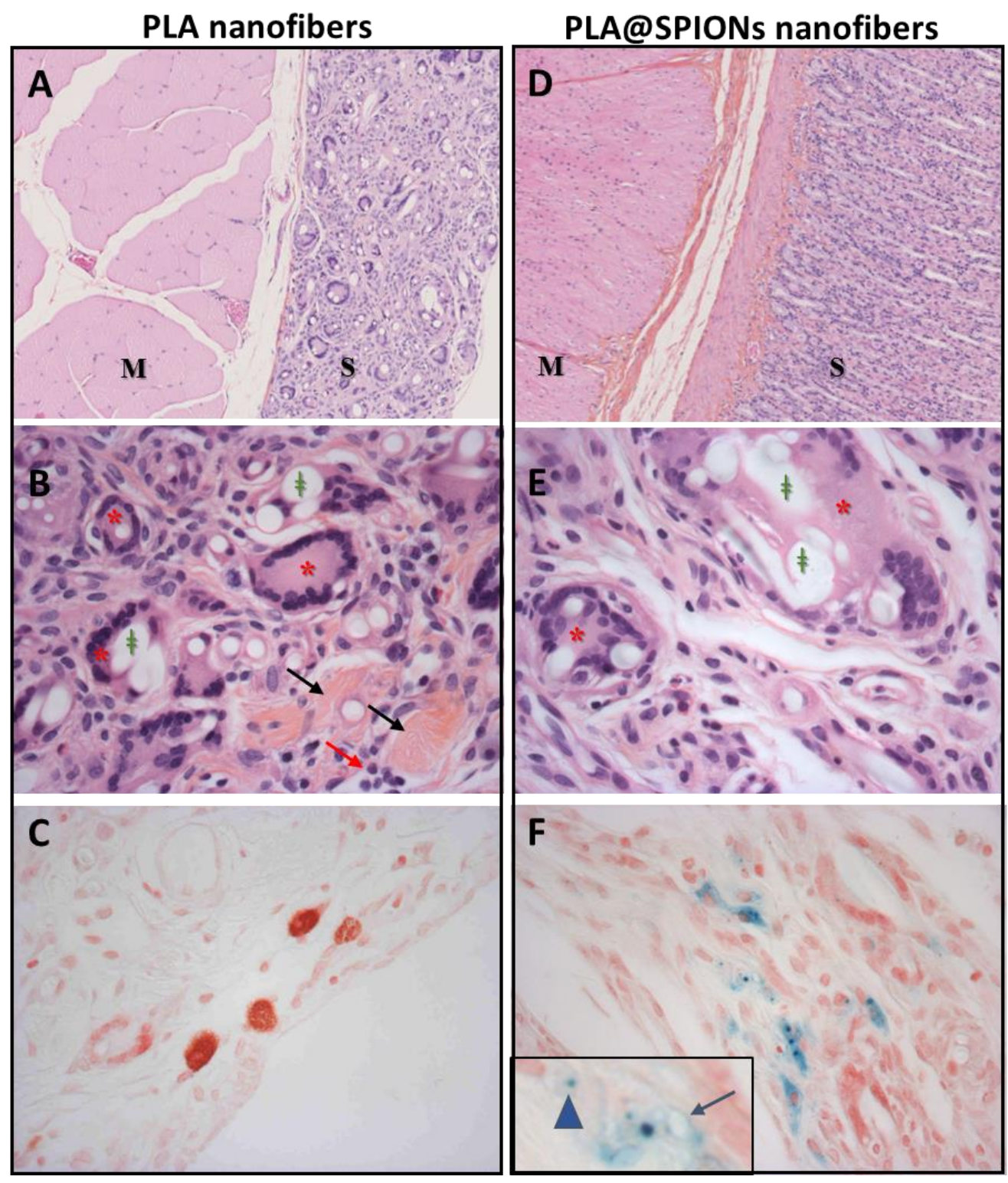

Figure 4. Host versus graft reaction after 3 months post-implantation of PLA (A to C) \& PLA@SPIONs (D to F) scaffolds. A\&B, D\&E are HES stained, C\&F are with Perls staining. A,D (5x magnification): the scaffold (S) and the abdominal muscle side (M) are juxtaposed. B,E (40x magnification): the immune cell infiltrate is composed of mononuclear cells (red arrow $\rightarrow$ ), macrophages and lymphocytes, and giant plurinucleated cells (red asterisk *) ; collagen fibers (black arrow $\rightarrow$ ) ; exogenous material (green double cross $\ddagger$ ). C,F (40x magnification): the cytoplasmic granular blue content is seen in the insert as a light blue film around the fibers in the giant plurinuclear cells (blue arrow $\rightarrow$ ) and a blue content in the cytoplasm of the macrophages (blue arrowhead $\mathbf{\Delta}$ ) for PLA@SPIONs scaffolds. 
At 6 months, the inflammatory reaction was still present without any noticeable difference between PLA and PLA@SPIONs scaffolds. On the HES stained slide sections, a moderate chronic inflammatory reaction of the foreign-body type was still observed for the PLA scaffolds (Figure 5A). The three cell types macrophages, lymphocytes and giant plurinucleated cells were present but in lower quantities than at 3 months. In contrast, mononuclear cells were increased with lymphocytes being sometimes multifocally grouped into clusters (Figure 5B). Macrophages and multinucleated giant cells responsible for the initial degradation of the nanofibers and clearing of the material, were therefore replaced by lymphocytes at longer time points. A careful analysis showed that multinucleated giant cells and nanofibers observed at 3 months in the cytoplasm of the giant cells were absent in the center of these clusters at 6 months. Around these clusters, giant multinucleated cells with nanofibers in the cytoplasm remained and some lymphocytes were concentrated near fine vessels, passing through the wall (Figure 5B). When present eosinophils were still associated with mast cells. Fibrosis was mildly increased.

The same moderate chronic inflammatory reaction developed against the nanocomposite scaffolds was observed for PLA@SPIONS after 6 months of implantation (Figure 5D). Like at 3 months, the comparison of the quantification and distribution of the cells showed similar results for the PLA@SPIONs fibers compared to the PLA fibers (Figure 5E and Table S2, supplementary data). After 6 months of implantation, the Perls staining confirmed the trend found at 3 months. No coloration was observed with the PLA fibers (Figure 5C), while a deep blue content in the cytoplasm of focally concentrated macrophages involved in the degradation process of the PLA@SPIONs scaffolds and the clearing of the SPIONs was visible in the case of the nanocomposite fibers (Figure 5F). 
Overall, this set of histological data highlights a moderate chronic inflammatory reaction that is similar for both PLA and PLA@SPIONs nanofibers. Thanks to Perls staining, it also reveals for the PLA@SPIONs scaffolds that the number of macrophages containing intracytoplasmic granulations and the intensity of blue staining increased at 6 months compared to 3 months, in accordance with their implication in the degradation of the scaffolds and in the elimination of the iron oxide nanoparticles, as reported for other magnetic biomaterials containing scaffolds. ${ }^{28}$ Beside this point, it is important to note the similar behaviors observed for the implanted PLA and PLA@SPIONs scaffolds. This was not the case for PCL nanofibers embedding SPIONs that showed a much faster degradation, resulting in a rapid rarefaction of the scaffold and a replacement by connective tissues, compared to pure PCL nanofibers over an 8 -week period. ${ }^{8}$ This confirms our initial hypothesis that the herein proposed strategy allows obtaining magnetic nanocomposites without alteration of the properties of the nanofibers, in this case degradation. Another main advantage of our approach compared to the classical electrospinning of polymer/SPIONs solutions to yield magnetic scaffolds is highlighted by the Perls staining. Indeed, whereas large and spread blue areas are observed all over the scaffold in the first case, our PLA@SPIONs only led to limited pale blue coloration as a result of the limited but sufficient amount of SPIONs required to yield a clear MRI visualization. ${ }^{6}$ This last point may be of advantage considering the recent reports on potential toxicity induced by SPIONs and their fate in the body. ${ }^{29,30}$ 


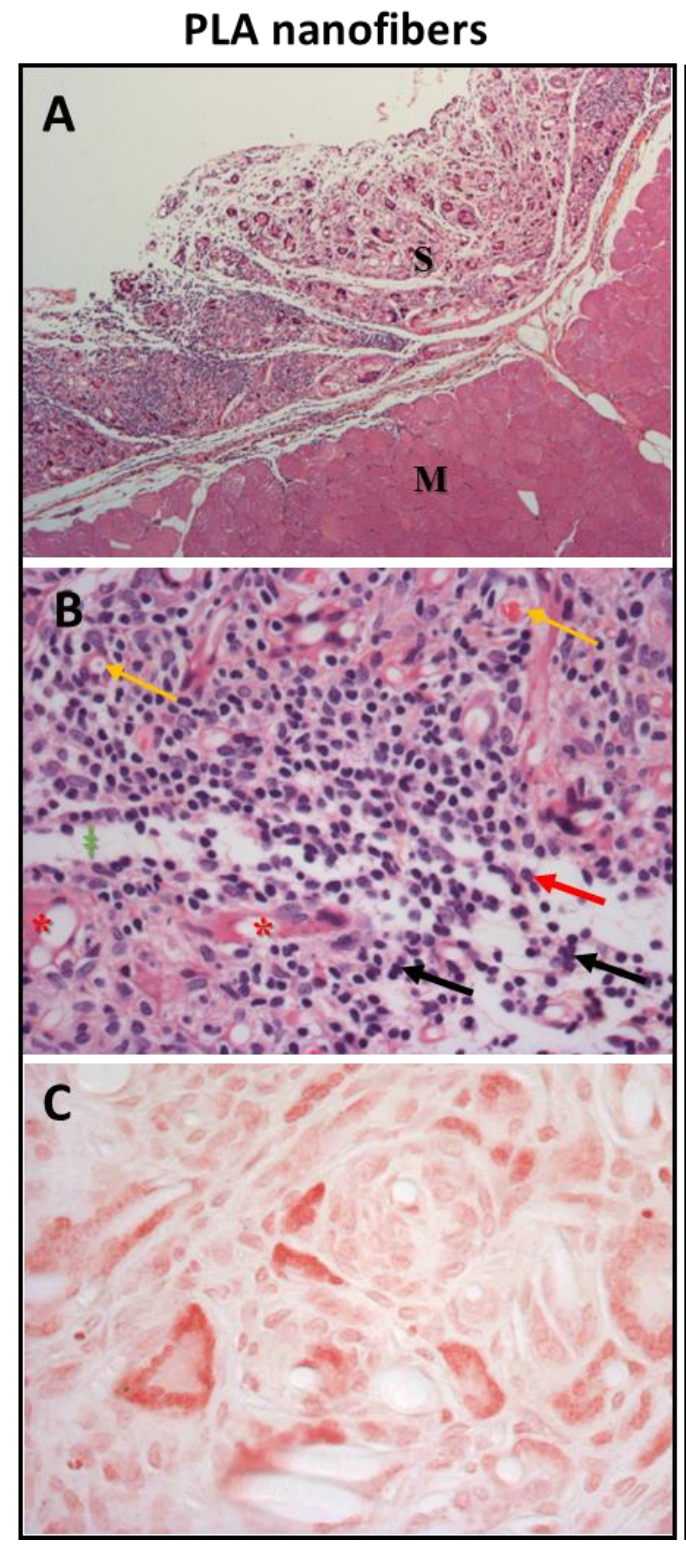

\section{PLA@SPIONs nanofibers}

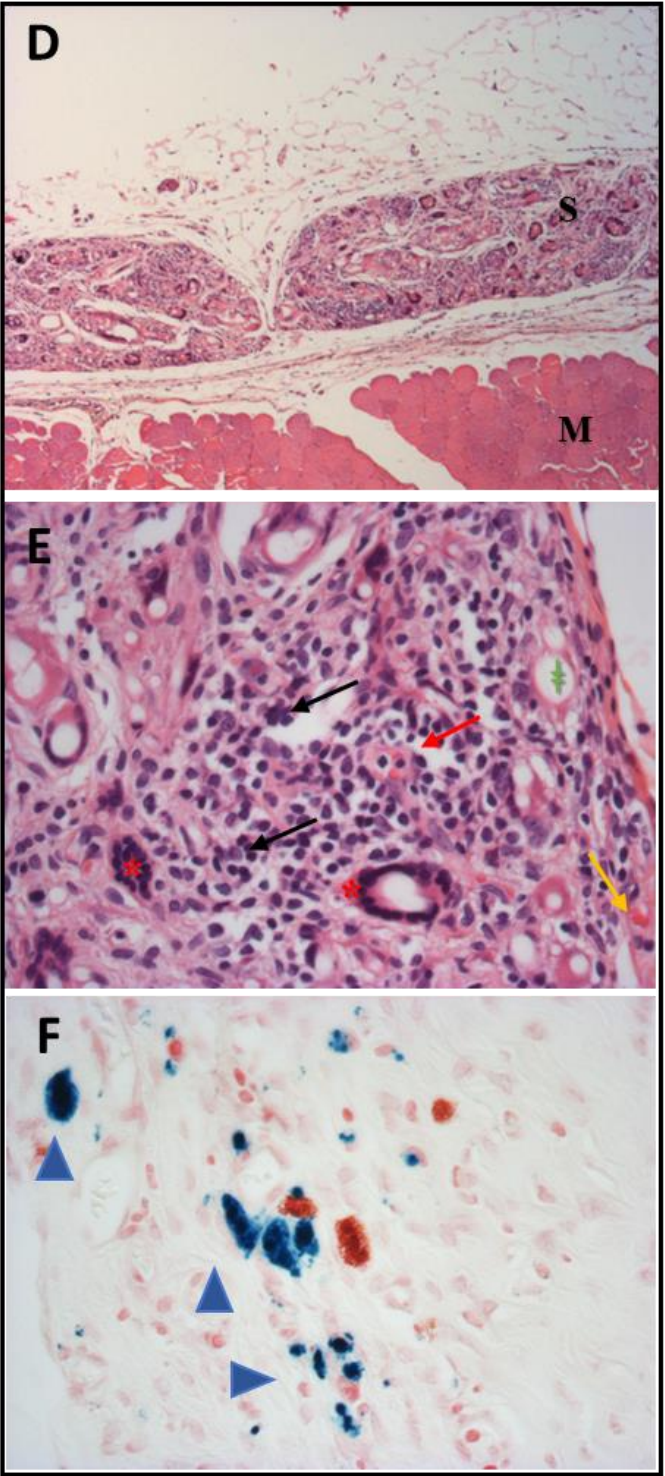

Figure 5. Host versus graft reaction after 6 months post-implantation of PLA and PLA@SPIONs scaffolds.

A-B \& D-E are HES stained, C\&F are with Perls staining. A, D (5x magnification): the scaffold (S) and the abdominal muscle side (M) are juxtaposed. B, E (40x magnification): the immune cell infiltrate is enriched of mononuclear cells (red arrow $\rightarrow$ ) sometimes multifocally grouped into clusters (black arrows $\rightarrow$ ), macrophages and lymphocytes, and giant plurinucleated cells are few (red asterisk *) ; vessels (orange arrows $\rightarrow$ ) ; exogenous material (green double cross ‡). C, F (40x magnification): a deep blue staining corresponding to the accumulated iron positive macrophages (blue arrowhead $\boldsymbol{\Delta}$ ) is seen for the PLA@SPIONs. 


\section{Conclusions}

We had recently reported on a procedure allowing for the preparation of PLA@SPIONs nanocomposites composed of PLA nanofibers covered by a quasi-monolayer of anchored SPIONs. ${ }^{16}$ In this work, we demonstrate that a rearrangement and redistribution of the SPIONs is observed around the PLA fibers upon in vitro degradation in PBS, as a result of the competition between the anchoring phosphonic groups and the phosphates coming from the simulated physiological medium at the surface of the SPIONs. However, we show that this does not impede the long-term in vivo MRI visualization of the PLA@SPIONs, that can be easily detected and imaged up to 6 months using clinically relevant MRI conditions. We also show similar integration of both PLA and PLA@SPIONs scaffolds, that trigger classical foreign body reactions of similar intensities, and we highlight the role of multinucleated giant cells and macrophages in the degradation process of these nanofibrous scaffolds and in the elimination of the iron oxide nanoparticles. This first insight in the tissue integration of PLA@SPIONs scaffolds could be further completed with flow cytometry experiments to quantify the immune response. Overall, this study proves that our PLA@SPIONs nanocomposites are just as well supported by the organism as bare PLA, and can therefore be suitable magnetic biomaterials for biomedical and tissue engineering applications. Future work will focus on the use of PLA@SPIONs nanofibers for biomedical applications and on the optimization of the fibers diameter, pores size and fibers organization (random, aligned etc...) to meet the requirements of each application.

CRediT author statement: Hussein Awada: Investigation, Writing-original draft. Saad Sene: Investigation. Danielle Laurencin: Conceptualization, Writing - review \& editing, Funding acquisition. Laurent Lemaire: Investigation, Methodology, Writing-original draft. Florence Franconi: Investigation, Methodology, Writing-original draft. Florence Bernex: Investigation, Writing-original draft. Audrey 
Bethry: Investigation. Xavier Garric: Visualization. Yannick Guari: Supervision, Funding acquisition. Benjamin Nottelet: Conceptualization, Writing-original draft, Writing - Review \& Editing, Funding acquisition, Supervision, Project administration.

\section{Conflicts of interest}

All contributing authors have no conflicts of interest to disclose.

\section{Acknowledgements}

Funding for this work was provided by the following sources: France Life Imaging FLI (ANR11-INBS-0006 grant from the French "Investissements d'Avenir" program) and LabEx CheMISyst (ANR-10-LABX-05-01 grant from the French "Investissements d'Avenir" program). Bertrand Rebière (ICGM) is acknowledged for his assistance in some of the SEM analyses presented herein, and Lucie Koeller from AETE-ISO platform, OSUOREME/Université de Montpellier for her assistance in the ICP analyses.

\section{References}

1 W. Park, H. Shin, B. Choi, W.-K. Rhim, K. Na and D. Keun Han, Prog. Mater. Sci., 2020, 114, 100686.

2 E. J. Bealer, K. Kavetsky, S. Dutko, S. Lofland and X. Hu, Int. J. Mol. Sci., 2019, 21, 186.

3 B. Sivakumar, R. G. Aswathy, R. Romero-Aburto, T. Mitcham, K. A. Mitchel, Y. Nagaoka, R. R. Bouchard, P. M. Ajayan, T. Maekawa and D. N. Sakthikumar, Biomater. Sci., 2017, 5, 432-443.

4 J. Keyvan Rad, Z. Alinejad, S. Khoei and A. R. Mahdavian, ACS Biomater. Sci. Eng., 2019, 5, 4425-4434. 
C. J. Mortimer and C. J. Wright, Biotechnol J., 2107, 12, 1600693.

6 J. Meng, B. Xiao, Y. Zhang, J. Liu, H. D. Xue, J. Lei, H. Kong, Y. G. Huang, Z. Y. Jin, N. Gu and H. Y. Xu, Sci. Rep.,2013, 3, 2655.

7 D. Y. Shan, Y. Z. Shi, S. Duan, Y. Wei, Q. Cai and X. P. Yang, Mater. Sci. Eng. C. 2013, 33, 3498-3505.

8 R. K. Singh, K. D. Patel, J. H. Lee, E. J. Lee, J. H. Kim, T. H. Kim and H. W. Kim, Plos One, 2014, 9, e91584.

9 A. I. Gonçalves, M. T. Rodrigues, P. P. Carvalho, M. Bañobre-López, E. Paz, P. Freitas and M. E. Gomes, Adv. Healthcare Mater., 2016, 5, 213-222.

10 Y. J. Kim, M. Ebara and T. Aoyagi, Adv. Funct. Mater., 2013, 23, 5753-5761.

11 C. Huang, S. J. Soenen, J. Rejman, J. Trekker, L. Chengxun, L. Lagae, W. Ceelen, C. Wilhelm, J. Demeester and S. C. De Smedt, Adv. Funct. Mater., 2012, 22, 2479-2486.

12 Z. Zhang, C. J. R. Wells, A. M. King, J. C. Bear, G.-L. Davies and G. R. Williams, J. Mater. Chem. B, 2020, 8, 7264-7274.

13 J. C. Fu, Q. F. Wang and J. B. Schlenoff, ACS Appl. Mater. Interfaces, 2015, 7, 895901.

14 J. O. Mangual, S. Li, H. J. Ploehn, A. D. Ebner and J. A. Ritter, J. Magn. Magn. Mater., 2010, 322, 3094-3100.

15 H. J. Lee, S. J. Lee, S. Uthaman, R. G. Thomas, H. Hyun, Y. Y. Jeong, C. S. Cho and I. K. Park, Int. J. Mol. Sci., 2015, 16, 13661-13677.

16 H. Awada, A. Al Samad, D. Laurencin, R. Gilbert, X. Dumail, A. El Jundi, A. Bethry, R. Pomrenke, C. Johnson, L. Lemaire, F. Franconi, G. Félix, J. Larionova, Y. Guari and B. Nottelet, ACS Appl. Mater. Interfaces, 2019, 11, 9519-9529.

17 M. E. Mertens, S. Koch, P. Schuster, J. Wehner, Z. Wu, F. Gremse, V. Schulz, L. Rongen, F. Wolf, J. Frese, V. N. Gesché, M. van Zandvoort, P. Mela, S. Jockenhoevel, F. Kiessling and T. Lammers, Biomaterials, 2015, 39, 155-163.

18 N. Kuehnert, N. A. Kraemer, J. Otto, H. C. W. Donker, I. Slabu, M. Baumann, C. K. Kuhl and U. Klinge, Surg. Endosc., 2012, 26, 1468-1475.

19 W. W. Yu, J. C. Falkner, C. T. Yavuz and V. L. Colvin, Chem. Commun., 2004, 23062307.

20 D. K. Balakrishnan-nair, N. D. Nair, S. K. Venugopal, V. N. Das, S. George, M. J. Abraham, S. Eassow, M. R. Alison, A. Sainulabdeen and T. V. Anilkumar, Toxicol. Pathol., 2018, 46, 169-183.

21 S. A. Grant, C. R. Deeken, S. R. Hamilton, D. A. Grant, S. L. Bachman and B. J. Ramshaw, J. Biomed. Mater. Res., Part A, 2013, 101, 2778-2787.

22 G. Guerrero, J. G. Alauzun, M. Granier, D. Laurencin and P. H. Mutin, Dalton Trans., 2013, 42, 12569-12585. 
23 J. C. Dias, C. Ribeiro, V. Sencadas, G. Botelho, J. L. G. Ribelles and S. LancerosMendez, Polym. Test., 2012, 31, 770-776.

24 J. Amalric, P. H. Mutin, G. Guerrero, A. Ponche, A. Sotto and J.-P. Lavigne, J. Mater. Chem., 2009, 19, 141-149.

25 A. Tzameret, H. Ketter-Katz, V. Edelshtain, I. Sher, E. Corem-Salkmon, I. Levy, D. Last, D. Guez, Y. Mardor, S. Margel and Y. Rotenstrich, J. Nanobiotechnol., 2019, 17, DOI:10.1186/s12951-018-0438-y.

26 A. Leftin and J. A. Koutcher, Contrast Media Mol. Imaging, 2018, 2018, 1-9.

27 E. Girard, G. Chagnon, A. Broisat, S. Dejean, A. Soubies, H. Gil, T. Sharkawi, F. Boucher, G. S. Roth, B. Trilling and B. Nottelet, Acta Biomater., 2020, 106, 70-81.

28 S. B. Campbell, M. Patenaude and T. Hoare, Biomacromolecules, 2013, 14, 644-653.

29 S. Ghosh, I. Ghosh, M. Chakrabarti and A. Mukherjee, Food Chem. Toxicol., 2020, 136, 110989.

30 V. Frantellizzi, M. Conte, M. Pontico, A. Pani, R. Pani and G. De Vincentis, Nucl. Med. Mol. Imaging, 2020, 54, 65-80. 